\title{
ESTILO DE APRENDIZAJE Y DESEMPEÑO ACADÉMICO: VARIABLES DEL ENTORNO EDUCATIVO
}

\author{
Jhon Harol Gonzáles Garay y Josué E. Turpoa \\ Universidad Peruana Unión, Lima - Perú \\ Recibido: 22 de abril de 2017 Aceptado: 18 de mayo de 2017
}

\begin{abstract}
Resumen
El objetivo del artículo es determinar la relación que existe entre los estilos de aprendizaje y el desempeño académico en los estudiantes de la Escuela Profesional de Arquitectura de la Universidad Peruana Unión Filial Tarapoto, 2017. La metodología corresponde al diseño correlacional transeccional con aplicación de instrumentos validados en una población de estudiantes de arquitectura. Entre los resultados se encontró que los estilos de aprendizaje se relacionan significativamente ( $\mathrm{p}$ value=0.05) con el desempeño académico en los estudiantes de Arquitectura de la Universidad Peruana Unión, Filial Tarapoto, 2017, de forma similar ocurre con la dimensión estilo teórico ( $r=-.193 * ; p=$ $.04)$; la dimensión estilo pragmático ( $r=-.259 * * ; p=.01)$, con el desempeño académico, sin embargo, el estilo reflexivo no se relaciona significativamente (p_value>0.05), así como la dimensión estilo activo no se relaciona sigñificativamente (p_value>0.05) con el desempeño académico en los estudiantes de Arquitectura de la Universidad Peruana Unión, Filial Tarapoto, 2017, por lo tanto son dos dimensiones con relación significativa son: estilo teórico y estilo pragmático, y dos dimensiones que no se relacionan que son: estilo reflexivo y estilo activo. En conclusión, se encuentra que los estilos de aprendizaje se relacionan significativamente (p_value=0.05) con el desempeño académico en los estudiantes de Arquitectura de la Universidad Peruana Unión, Filial Tarapoto, 2017.
\end{abstract}

Palabras clave: Estilos de aprendizaje; desempeño académico; educación universitaria; estudiantes de arquitectra

\section{Abstract}

The aim of this paper is to determine the relationship between learning styles and academic performance in the students of the School of Architecture at the Universidad Peruana Unión, Filial Tarapoto, 2017. It is a transactional correlational design with an application of validated instruments in a population of architecture students. Among the results it was found that the learning styles are significantly related ( $p_{\text {_value }}=0.05$ ) with the academic performance in the students of the School of Architecture at the Universidad Peruana Unión, Filial Tarapoto, 2017, similarly occurs with the theoretical style dimension $(r=-.193 * ; \mathrm{p}=.04)$; the pragmatic style dimension $(r=-.259 * * ; \mathrm{p}$ $=.01)$, with academic performance, however, the reflective style is not significantly related (p_value> 0.05), as well as the active style dimension is not significantly related ( $p$ value $>0.05$ ) with the academic performance of Architecture students of Universidad Peruana Unión, Filial Tarapoto, 2017, therefore, two dimensions with a significant relationship are: theoretical style and pragmatic style, and two dimensions that are not related to They are: reflective style and active style. In conclusion, it is found that the learning styles are 
Revista de Investigación Universitaria | Volumen 6 - Número 2, Julio - Diciembre, 2017 | DOI ISSN 2078-4015 (En línea)

significantly related ( $\mathrm{p}$ value $=0.05$ ) with the academic performance of Architecture students of the Universidad Peruana Unión, Filial Tarapoto, 2017.

Palabras clave: Learning styles, academic performance, University education, architecture students 
Revista de Investigación Universitaria | Volumen 6 - Número 2, Julio - Diciembre, 2017 | DOI

\section{Introducción}

Los diferentes paradigmas educativos describen a los estudiantes como procesadores activos de la información, es el estudiante quien genera su propio conocimiento y se convierte finalmente en gestor de su aprendizaje (Garret Vargas, 2013a). Frente a ello, los diferentes cambios acelerados que involucran a los agentes educativos, sea el estudiante, sea el maestro están inmersos en este rápido conocimiento y especialización.

Vivimos en una época donde el conocimiento y la información se hace cada vez más accesible a través de las redes de internet, y la necesidad de tener modelos de enseñanza y aprendizaje basados en la transmisión y almacenamiento de conocimiento pierden su funcionalidad. Dentro del sistema educativo convencional existen estudiantes que a menudo adquieren un cierto conocimiento porque simplemente están siendo probados y no porque responden a propósitos educativos (Mara, 2010).

Sin embargo, la sociedad exige desarrollar la capacidad de los estudiantes para actualizar sus conocimientos siempre que sea necesario. Asimismo, la competitividad académica en estos últimos años se ha ido incrementado cada vez más, es así que; actualmente "las exigencias de una preparación académica, para afrontar los retos de la vida universitaria debe ser óptima, prioritaria y significativa, pues las implicancias de la vida universitaria suelen a veces ser para algunos atractiva, emocionante, nueva y excitante, mientras que para otros se torna difícil, ansiosa y con mayor presión que en la secundaria" (Carranza, 2015 p. 12).

Se han realizado diferentes estudios sobre los estilos de apredizaje y estilos cognitivos (Bahamón Muñetón, Vianchá Pinzón, Alarcón Alarcón, \& Bohórquez Olaya, 2012). Asimismo, el objetivo de estos estilos es desarrollar en los 
Revista de Investigación Universitaria | Volumen 6 - Número 2, Julio - Diciembre, 2017 | DOI

estudiantes el aprendizaje independiente, en los cuales el estudiante seleccione y elabore sus propias concepciones. Para ello el docente es clave en la selección de estrategias adecuadas para enseñar a los alumnos a aprender (Fernández \& Beligoy, 2015).

\section{Materiales y métodos}

El método sigue el tipo correlacional, porque permite estudiar la relación que existe entre los estilos de aprendizaje y el desempeño académico de los estudiantes de la Escuela Profesional de Arquitectura (Hernández, Fernández y Baptista, 2006). El presente estudio, responde a un diseño no experimental de corte transversal, dado que no habrá manipulación de variables y los datos serán recolectados en un momento único.

\subsection{Instrumentos}

El método de recolección de datos será la encuesta y los Instrumentos utilizados son el Cuestionario Honey-Alonso de Estilos de Aprendizaje (CHAEA), en donde está incluida información sobre datos demográficos tales como el género, edad, procedencia y ciclo de estudios. Para este momento ya se tendrá el Instrumento de medición previamente adaptado.

Posteriormente teniendo la autorización para llevar a cabo la aplicación de los Instrumentos, se dará las debidas instrucciones sobre cómo desarrollar el cuestionario, y también se creará un clima agradable a fin de captar la atención sobre la aplicación del instrumento y cómo les beneficiará según los resultados obtenidos. Al finalizar la aplicación, se le agradecerá por su participación y colaboración.

El cuestionario de estilos de aprendizaje (CHAEA) de Honey y Alonso contiene 80 ítems distribuidos en 4 dimensiones: Estilo de aprendizaje reflexivo, teórico, pragmático y activo; deben ser respondidos a través de dos opciones de 
Revista de Investigación Universitaria | Volumen 6 - Número 2, Julio - Diciembre, 2017 | DOI

respuesta cerrada $(+y-)$ donde la persona debe marcar según su grado de preferencia según sea sus estilos de aprendizaje.

El cuestionario CHAEA ha demostrado adecuada validez y confiabilidad en diversos estudios (Honey y Mumford, 1986; Alonso, Gallego \& Honey, 1995; Ruiz, 2004; Prieto, 2010, Díaz, 2010; Tatau, 2011; Ferrón, 2012 y Varela, 2014).

Respecto a la variable desempeño académico, se tomará como referencia el promedio de las notas (ponderado) obtenidas por los estudiantes de la Escuela Académico Profesional de Arquitectura de la Universidad Peruana Unión.

\section{Resultados y Discusión}

A continuación, se presentan los resultados obtenidos en el presente estudio.

\subsection{Resultados 1}

Se observa en la tabla 01 que el estilo de aprendizaje con mayor predominancia es el estilo teórico (37.5\%) seguido del estilo pragmático (29.5\%). En cuanto a la preferencia moderada, se percibe que el estilo reflexivo y activo son puestos en práctica por los estudiantes de la Escuela Profesional de Arquitectura de la Universidad Peruana Unión Filial Tarapoto (47.3 y 43. ㅇ respectivamente).

Tabla 01

Estilos de aprendizaje de los estudiantes de Arquitectura de la Universidad Peruana Unión Filial Tarapoto

\begin{tabular}{llll}
\hline $\begin{array}{l}\text { Estilos de } \\
\text { aprendizaje }\end{array}$ & Niveles & $\mathrm{n}$ & \multicolumn{1}{c}{} \\
\hline Estilo activo & Muy baja & 3 & $2.7 \%$ \\
& Baja & 5 & $4.5 \%$ \\
& $\begin{array}{l}\text { Preferencia } \\
\text { moderada }\end{array}$ & 49 & $43.8 \%$ \\
& Alta & 32 & $28.6 \%$ \\
& Muy alta & 23 & $20.5 \%$ \\
Estilo & Muy baja & 11 & $9.8 \%$ \\
reflexivo & Baja & 19 & $17.0 \%$ \\
\hline
\end{tabular}




\begin{tabular}{llll}
\hline & $\begin{array}{l}\text { Preferencia } \\
\text { moderada }\end{array}$ & 53 & $47.3 \%$ \\
Alta & 26 & $23.2 \%$ \\
Muy alta & 3 & $2.7 \%$ \\
Estilo teórico & Muy baja & 0 & $0.0 \%$ \\
& Baja & 9 & $8.0 \%$ \\
& Preferencia & 28 & $25.0 \%$ \\
moderada & 33 & $29.5 \%$ \\
Alta & Muy alta & 42 & $37.5 \%$ \\
Mutilo & 4 & $3.6 \%$ \\
& Baja baja & 9 & $8.0 \%$ \\
& Preferencia & 34 & $30.4 \%$ \\
moderada & & \\
Alta & 32 & $28.6 \%$ \\
& Muy alta & 33 & $29.5 \%$ \\
& Total & 112 & $100.0 \%$ \\
\hline
\end{tabular}

\subsection{Resultados 2}

En la tabla 02 se aprecia que tanto hombres como mujeres practican moderadamente los cuatro estilos de aprendizaje (Estilo activo 23.2\% y 20.5\%; Estilo reflexivo 28.6\% y $18.8 \%$; Estilo teórico $18.8 \%$ y $6.3 \%$; Estilo pragmático $18.8 \%$ y $11.6 \%)$.

Tabla 02

\begin{tabular}{|c|c|c|c|c|c|}
\hline \multirow[t]{3}{*}{ Variable } & \multirow[t]{3}{*}{ Niveles } & \multicolumn{4}{|c|}{ Genero } \\
\hline & & \multicolumn{2}{|c|}{ Masculino } & \multicolumn{2}{|c|}{ Femenino } \\
\hline & & $\mathrm{n}$ & 응 & $\mathrm{n}$ & 응 \\
\hline \multirow{6}{*}{$\begin{array}{l}\text { Estilo } \\
\text { activo }\end{array}$} & Muy baja & 2 & $1.8 \%$ & 1 & $.9 \%$ \\
\hline & Baja & 5 & $4.5 \%$ & 0 & $0.0 \%$ \\
\hline & $\begin{array}{l}\text { Preferencia } \\
\text { moderada }\end{array}$ & 26 & $23.2 \%$ & 23 & $20.5 \%$ \\
\hline & Alta & 25 & $22.3 \%$ & 7 & $6.3 \%$ \\
\hline & Muy alta & 14 & $12.5 \%$ & 9 & $8.0 \%$ \\
\hline & Muy baja & 8 & $7.1 \%$ & 3 & $2.7 \%$ \\
\hline
\end{tabular}




\begin{tabular}{llllll}
\hline $\begin{array}{l}\text { Estilo } \\
\text { reflexivo }\end{array}$ & Baja & 13 & $11.6 \%$ & 6 & $5.4 \%$ \\
& $\begin{array}{l}\text { Preferencia } \\
\text { moderada }\end{array}$ & 32 & $28.6 \%$ & 21 & $18.8 \%$ \\
& Alta & 16 & $14.3 \%$ & 10 & $8.9 \%$ \\
& Muy alta & 3 & $2.7 \%$ & 0 & $0.0 \%$ \\
Estilo & Muy baja & 0 & $0.0 \%$ & 0 & $0.0 \%$ \\
teórico & Baja & 6 & $5.4 \%$ & 3 & $2.7 \%$ \\
& $\begin{array}{l}\text { Preferencia } \\
\text { moderada }\end{array}$ & 21 & $18.8 \%$ & 7 & $6.3 \%$ \\
& Alta & 20 & $17.9 \%$ & 13 & $11.6 \%$ \\
Estilo & Muy alta & 25 & $22.3 \%$ & 17 & $15.2 \%$ \\
Mragmático baja & 3 & $2.7 \%$ & 1 & $.9 \%$ \\
& Baja & 7 & $6.3 \%$ & 2 & $1.8 \%$ \\
& $\begin{array}{l}\text { Preferencia } \\
\text { moderada }\end{array}$ & 21 & $18.8 \%$ & 13 & $11.6 \%$ \\
& Alta & 17 & $15.2 \%$ & 15 & $13.4 \%$ \\
& Muy alta & 24 & $21.4 \%$ & 9 & $8.0 \%$ \\
& Total & 72 & $64.3 \%$ & 40 & $35.7 \%$ \\
\hline
\end{tabular}

\subsection{Resultados 3}

\subsubsection{Technical analysis}

En la Tabla 9 se observa que el estilo teórico se relaciona significativamente con el desempeño académico ( $r=-.193 *$; $\mathrm{p}=.04)$, así también el estilo pragmático se relaciona significativamente con el desempeño académico ( $r=-.259 *$; $\mathrm{p}=.01)$. Por otro lado, se aprecia que no existe relación significativa entre el estilo activo, reflexivo y desempeño académico.

Tabla 03

Coeficientes de correlación entre estilos de aprendizaje y desempeño académico.

\begin{tabular}{lll}
\hline Estilos de & \multicolumn{2}{l}{ Desempeño académico } \\
\cline { 2 - 3 } aprendizaje & Rho & p \\
Estilo activo &.-.118 & .216 \\
Estilo reflexivo & -.104 & .275 \\
Estilo teórico & $-.193 \star$ & .042 \\
Estilo pragmático & $-.259 \star \star$ & .006 \\
\hline$\star \star$ La correlación es & significante a nivel 0.01 \\
\hline
\end{tabular}




\section{Conclusiones}

El propósito de este estudio fue evaluar la relación que existe entre los estilos de aprendizaje y el desempeño académico en los estudiantes de la Escuela Profesional de Arquitectura de la Universidad Peruana Unión Filial Tarapoto, 2017.

Los resultados del estudio indican que los estilos de aprendizaje se relacionan significativamente (p_value=0.05) con el desempeño académico en los estudiantes de Arquitectura de la Universidad Peruana Unión, Filial Tarapoto, 2017, de forma similar ocurre con la dimensión estilo teórico ( $r$ = .193*; $\mathrm{p}=.04)$; la dimensión estilo pragmático ( $r=-.259 *$; $\mathrm{p}=.01)$, con el desempeño académico, sin embargo, el estilo reflexivo no se relaciona significativamente (p_value>0.05), así como la dimensión estilo activo no se relaciona significativamente ( $p_{-}$value>0.05) con el desempeño académico en los estudiantes de Arquitectura de la Universidad Peruana Unión, Filial Tarapoto, 2017, por lo tanto son dos dimensiones con relación significativa son: estilo teórico y estilo pragmático, y dos dimensiones que no se relacionan que son: estilo reflexivo y estilo activo.

Esto indica que estas dimensiones influyen positivamente, si su valor aumenta ambas lo hacen. Mediante los estilos de aprendizaje se observa una orientación positiva hacia el desempeño académico, esto es consistente con lo reportado por Acevedo, Cavadia y Alvis (2015). Asimismo, estos resultados están en línea por lo investigado por Casani García (2013), Chapilliquen y Purihuaman (2013).

En cuanto a los resultados sobre las dimensiones estilo reflexivo y estilo teórico que no correlacionan, resultados similares se encuentran por lo reportado por OrtizFernández, et al (2016), sin embargo, es diferente a lo 
Revista de Investigación Universitaria | Volumen 6 - Número 2, Julio - Diciembre, 2017 | DOI

reportado por Alducin-Ochoa y Vásquez-Martínez (2015) que menciona que existe un predominio del estilo reflexivo.

Los resultados de este estudio además revelan que los estilos de aprendizaje con mayor predominancia son el estilo teórico (37.5\%) seguido del estilo pragmático (29.5\%). En cuanto a la preferencia moderada, se percibe que el estilo reflexivo y activo son puestos en práctica por los estudiantes de la Escuela Profesional de Arquitectura de la Universidad Peruana Unión Filial Tarapoto (47.3\% y 43 . 응 respectivamente).

Asimismo, se observa en cuanto a género que tanto hombres como mujeres practican moderadamente los cuatro estilos de aprendizaje (Estilo activo 23.2\% y 20.5\%; Estilo reflexivo $28.6 \%$ y 18.8\%; Estilo teórico 188\% y 6.3\%; Estilo pragmático 18.8\% y 11.6\%). En relación a la muestra se observa que el 89.6\% de estudiantes de la Escuela Profesional de Arquitectura se ubica en un nivel promedio respecto al desempeño académico.

Aunque se trabajó con instrumentos estandarizados es probable que en su aplicación a la muestra seleccionada hay existido falencias, sin embargo, la incorporación de soluciones para mejorar los resultados está acorde a lo trabajado por Purihuaman (2013).

La revisión del estado del arte otorga mucha información sobre las variables estudiadas, sin embargo, este estudio es aplicado en una institución confesional con un amplio énfasis en los aspectos reflexivos.

El presente estudio, con un diseño descriptivo correlacional adecuado y con una muestra representativa, encuentra múltiples factores que deben ser considerados para futuros estudios.

Diversos autores corroboran largamente los conceptos de relación entre los estilos de aprendizaje y el desempeño 
Revista de Investigación Universitaria | Volumen 6 - Número 2, Julio - Diciembre, 2017 | DOI

académico. Además, mencionan que existe una relación positiva entre las dimensiones de estilos de aprendizaje y desempeño académico (Demirbaş Osman, 2001b; Olivos et al., 2016; Pantoja Ospina, Duque Salazar, \& Correa Meneses, 2013)

\section{Referencias}

Acevedo, D., Cavadia, S., Y Alvis, A. (2015). Estilos de aprendizaje de los estudiantes de la Facultad de Ingeniería de la Universidad de Cartagena (Colombia). Formación Universitaria, $8(4)$, $15-22$. https://doi.org/10.4067/s0718-50062015000400003

Alducin-Ochoa, J., y Vázquez-Martínez, A. (2016). Autoevaluación de Conocimientos Previos y Rendimiento según Estilos de Aprendizaje en un Grado Universitario de Edificación. Formación Universitaria, 9(2), 29-40. Retrieved from http://www.redalyc.org/articulo.oa?id=373544971004

Aragón-Jiménez, L. (2016). Marco teorico metodologico de la evaluacion para aprender. Calidad En La Educación Superior, $7(1), 100-126$.

Bahamón-Muñetón, M., Vianchá-Pinzón, M., Alarcón-Alarcón, L., y Bohórquez-Olaya, C. (2012). Estilos y estrategias de aprendizaje: una revisión empírica y conceptual de los últimos diez años. Pensamiento Psicológico, 10(1), 129144 .

Borracci, R., y Arribalzaga, E. (2015). Estilos de aprendizaje de Kolb en estudiantes de medicina. Medicina (Buenos Aires), 75(2), 73-80.

Casani-García, J. (2011). Relación entre los estilos de aprendizaje y el rendimiento académico de los alumnos de internado estomatológico profesional de Estomatología de la Universidad Alas Peruanas, Filial Arequipa, 2011. Universidad Católica de Santa María - UCSM. UCSM. Retrieved from

http://tesis.ucsm.edu.pe/repositorio/bitstream/handle/UCS M/4596/96.1087.MG.pdf? sequence=1\&isAllowed=y

Cascón, I. (2000). Análisis de las calificaciones escolares como criterio de rendimiento académico. In C. P. J. G. Pérez (Ed.), Jornadas Científicas (pp. 1-11). Salamanca, España: Universidad de Salamanca.

Cerón, S., y Limatorres, H. (2016). La coherencia constructivista como estrategia didactica para el aprendizaje. Educación Y Desarollo Social., 10(2), 50-85. 
Chain-Revuelta, R., Cruz-Ramírez, N., Martínez-Morales, M., y Jácome-Ávila, N. (2003). Examen de selección y probabilidad de éxito escolar en estudios superiores: Estudio en una universidad pública estatal mexicana. Revista Electrónica de Investigación Educativa, 5(1), 117. Retrieved from http://www.scielo.org.mx/scielo.php?script=sci_arttext\&pi $d=S 1607-40412003000100005 \& l n g=e s \& n r m=i s o \& t l n g=e s$

Chapilliquen-Rodríguez, M. (2016, April 20). Competencias digitales en estudiantes con diferentes estilos de aprendizaje, del séptimo ciclo de educación secundaria, desarrolladas a través de la red social educativa Edmodo en una institución educativa pública de la Unidad de Gestión Educativa Local N. Pontificia Universidad Católica del Perú. Retrieved from http://tesis.pucp.edu.pe/repositorio/handle/123456789/674 4

Defagó, C. (2012). Tras los rastros del lenguaje: revisión teórica del modelo de rr de karmiloff smith. Revista Argentina de Ciencias Del Comportamiento, 4(1), 44-55.

Del Alamo-Pantigoso, J. (2012). Relación entre desempeño docente y nivel del logro de aprendizaje de los oficiales alumnos de la maestria en Ciencias Militares. Universidad Nacional de Educación Enrique Guzman y Valle. Retrieved from

http://www.une.edu.pe/modelo_PROYECTO_CORRELACIONAL.pdf

Demirbaş Osman, Ö. (2001). The Relation of Learning Styles and Performance Scores of the students in Interior Architecture Education. Bilkent Üniversitesi. Bilkent Üniversitesi. Retrieved from http://www.thesis.bilkent.edu.tr/0001800.pdf

Fernández, V., y Beligoy, M. (2015). Estilos de aprendizaje y su relación con la necesidad de resestructuración de las estrategias de aprendizaje de los estudiantes universitarios de primer año. Fundación Educación Médica FEM, $\quad 18(5), \quad 361-366$. Retrieved from http://scielo.isciii.es/pdf/fem/v18n5/original8.pdf

Garret-Vargas, P. (2013a). Estilos de aprendizaje según sexo, facultad y ciclo de estudios en alumnos de un Instituto Superior Tecnológico de Lima. Pontificia Universidad Católica del Perú. Retrieved from http://tesis.pucp.edu.pe/repositorio/handle/123456789/169 9 
Harb, J., Durrant, S., y Terry, R. (1993). Use of the Kolb Learning Cycle and the $4 \mathrm{MAT}$ System in Engineering Education. Journal of Engineering Education, 82(2), 70-77.

Hernández-Serrano, M., Serate-González, S., y Campos-Ortuño, R. (2015). Influencia del estilo de aprendizaje y del tipo de tarea en los procesos de búsqueda en línea de estudiantes universitarios. Investigación Bibliotecológica, 29(65), 115-136.

Molina, O. (2012). El profesor ante la formacion de valores. aspectos teoricos y practicos. TESI, 13(3), 240-267.

Olivos, P., Santos, A., Martín, S., Cañas, M., Gómez-Lázaro, E., y Maya, Y. (2016). The relationship between learning styles and motivation to transfer of learning in a vocational training programme. Suma Psicológica, 23(1), 25-32. https://doi.org/10.1016/j.sumpsi.2016.02.001

Ortiz-Fernández, L., Moromi-Nakata, H., Gutiérrez-Ilave, M., Barra- Hinostroza, M., Cáceres de Barcés, L., Quintana del Solar, C., ... Guillén-Pariona, M. (2016). Estilos de aprendizaje y rendimiento académico en estudiantes ingresantes de Odontología. Odontología Sanmarquina, 18(2), 71. https://doi.org/10.15381/os.v18i2.11517

Pantoja-Ospina, M., Duque-Salazar, L., y Correa-Meneses, J. (2013). Learning Styles Models: An upgrade for their revision and analysis. Revista Colombiana de Educación, (64), 79-105.

Perales-Dávila, D., y Gonzales-Soto, T. (2014). Programa lúdico y aprendizajes matemáticos en el organizador números, relaciones y operaciones en Primaria. Universidad Cesar Vallejo, 3, 17-24.

Piaget, J. (1976). Teorías Del Aprendizaje, 1-9.

Purihuamán, C. (2013). Estilos de aprendizaje y rendimiento académico en estudiantes del I y II ciclo de Ingeniería Civil de la Universidad César Vallejo. TZHOECOEN, 5(1), 123-136. Retrieved from http://servicios.uss.edu.pe/ojs/index.php/tzh/article/vie w/56/55

Rettis-Salazar, H. (2015). Estilos de aprendizaje y rendimiento académico de la asignatura de estadística de los estudiantes del III ciclo de la EAPA, Facultad de Ciencias Administrativas - UNMSM - 2015. Tesis digitales - UNMSM. Universidad Nacional Mayor de San Marcos. Programa Cybertesis PERÚ. 
Reyes-Carreto, R., Godínez-Jaimes, F., Ariza-Hernández, F. J., Sánchez-Rosas, F., y Torreblanca-Ignacio, O. (2014). Un modelo empírico para explicar el desempeño académico de estudiantes de bachillerato. Perfiles Educativos, 36(146), 45-62. Retrieved from http://www.scielo.org.mx/pdf/peredu/v36n146/v36n146a4.pdf

Rojas, M. (2002). Aprendizaje transformacional en la familia y en la educación. Revista Venezolana de Análisis de Coyuntura, 8(1), 189-200.

Romero, L., Salinas, V., y Mortera, F. (2010). Estilos de aprendizaje basados en el modelo de Kolb en la educación virtual. Apertura, 1(2), 72-85.

Rondon, S., Rumbo, M., Arenas, A., Carvajal, S., Serna, C., Palacio, D., y Daza, A. (1979). Origen del Cognitivismo.

Salazar-Lugo, G. M., García-López, R. I., BalderramaTrápaga, J., \& Rodríguez-Torres, L. (2016). Estado afectivo en la identificación automática de estilos de aprendizaje. Apertura, 8(1), 1-15. Retrieved from http://www.redalyc.org/articulo.oa?id=68845366008

Subía-Arellano, A., y Gordón, J. (2014). Esbozo critico sobre las estructuras cognitivas: genesis del pensamiento cientifico. Colección De Filosofía De La Educación, 16(1), $71-82$.

Tripodoro, V., \& De Simone, G. (2015). Nuevos paradigmas en la educación universitaria. Los estilos de aprendizaje de David Kolb. Medicina, (75), 113-118.

Ventura, A. (2013). El ajuste instructivo entre estilos de aprendizaje y enseñanza en la universidad. Revista de Psicología, $31(2)$, 265-286. Retrieved from http: // search. ebscohost. com/login. aspx?direct=true\&db=zbh $\& A N=102928588 \&$ lang=es \&site $=e d s-l i v e$

Vielma, E., y Salas, M. (2000). Aportes de las teorías de Vygotsky, Piaget, Bandura y Bruner. Paralelismo en sus posiciones en relación con el desarrollo. Educere, 3(13164910), 30-37. https://doi.org/1316-4910

Zapata-Ros, M. (2015). Teorías y modelos sobre el aprendizaje en entornos conectados y ubicuos. Bases para un nuevo modelo teórico a partir de una visión crítica del "conectivismo." Education in the Knowledge Society (EKS), $16(1)$, 69-102. https://doi.org/10.14201/eks201516169102

Zin, N. A., Zaman, H. B., \& Noah, S. A. (2002). Multimedia Mathematics Tutor: Matching Instruction to student's Learning Styles. ICCE, 1433. 